pleural tissue at each time point and summarised as Mean $(+/-S D)$.

Pleural biopsies were obtained at MT in $8 / 9$ patients who underwent complete CE-MRI. Paraffin-embedded tissue was available for 6/8 and stained with Factor VIII and CD34 immunostains. Blood vessel numbers and total vessel area were measured using quantitative image-analysis software (Leica Biosystems, U. K.) and correlated against contrast kinetic parameters (early SI increment (0-4.5 min) and peak SI), using Spearman's test. Patients were followed-up in a specialist pleural clinic and survival recorded.

Results Mean age was 75 years $(+/-7) .93 \%(n=14)$ were male. Final diagnoses were: MPM $(\mathrm{n}=6)$, lung adenocarcinoma $(\mathrm{n}=1)$, breast adenocarcinoma $(\mathrm{n}=1)$, renal cell carcinoma $(\mathrm{n}$ $=1)$, Benign Asbestos Pleural Effusion $(\mathrm{n}=4)$, rheumatoid arthritis-related effusion $(n=1)$ and haemothorax $(n=1)$.

Figure 1 demonstrates relationships identified between contrast kinetic parameters and tissue vascularity. Mean follow-up was 267 (+/- 149) days, over which time mortality for MPM patients exhibiting early peak CE was $100 \%(\mathrm{n}=2 / 2)$ vs. $0 \%(\mathrm{n}$ $=0 / 1)$ for late peak CE $(\log$ rank $\mathrm{p}=0.2)$.

Conclusions We have established a functional MRI protocol for use in MPM. Within the limitations of this pilot study, early CE kinetics appear to reflect pleural tissue vascularity. Further work is ongoing to fully assess the diagnostic, prognostic and predictive value of this imaging biomarker.

\section{S45 HOW SUCCESSFUL ARE MEDICAL THORACOSCOPISTS AT PREDICTING MALIGNANCY?}

RJ Hallifax, JP Corcoran, I Psallidas, NM Rahman. Oxford Centre for Respiratory Medicine, Oxford, UK

\subsection{6/thoraxjnl-2014-206260.51}

Introduction Use of medical thoracoscopy by physicians to diagnose malignant pleural disease is increasing. Thoracoscopy is also used therapeutically to pleurodese (talc poudrage), at the time of biopsy, to minimise pleural effusion recurrence. However, this relies on the physician being confident of their diagnosis macroscopically.

A survey was conducted of regular thoracoscopists to establish the current practise related to medical thoracoscopy and a video of previous thoracoscopies (with known histology) was used to assess the accuracy of macroscopic evaluation of pleural disease. Methods 20 video clips recorded during thoracoscopy in Oxford were combined into a short video ( $30 \mathrm{~s}$ per clip): including a selection of cases with malignant (13) and benign disease (7), intentionally including some cases which were deemed "unclear". A survey of thoracoscopists was conducted via email. Respondents were asked to state whether each clip showed malignant or benign disease, scoring their confidence in their diagnosis (out of 10), whether they predict trapped lung and if they would perform pleurodesis. Gold standard of diagnosis was the histology result.

Results Procedural survey: 16 physicians responded from 12 tertiary referral centres: 13 consultants and 3 specialist registrars. 15 (94\%) had performed $>30$ thoracoscopies each. Four institutions $(25 \%)$ perform between $>10$ thoracoscopies per month; 12 (75\%) between 1-10 per month. Only 6 (38\%) perform thoracoscopies as day cases. All perform rigid rather than flexible thoracoscopies.

Video survey: Of the 20 video clips, the mean number of correct answers was 12.4 (62\%). Respondents were more confident of their answers (out of 10) when correct (7.1/10) than incorrect (6.1/10). In cases deemed malignant, 69\% would have performed talc pleurodesis; however, 17\% would have pleurodesed patients who had benign disease (See Table). Respondents only correctly predicted trapped lung in $2.6 / 20$ cases (13\%).

Conclusion Despite being experienced thoracoscopists, only $62 \%$ correctly diagnosed malignant or benign on video clips. The majority would appropriately perform pleurodesis, but $17 \%$ may have inappropriately pleurodesed benign disease. There are limitations to this small survey using short thoracoscopy clips, but this data suggests caution is required when considering making diagnosis on macroscopic appearance and deciding whether the lung is trapped.

\section{Basic mechanisms in COPD pathogenesis}

\section{S46 PHAGOCYTOSIS BY BLOOD NEUTROPHILS IS NOT ATTENUATED IN PATIENTS WITH CHRONIC OBSTRUCTIVE PULMONARY DISEASE}

GM Walton, T Purvis, C Chadwick, RA Stockley, E Sapey. University of Birmingham, Birmingham, UK

\subsection{6/thoraxjnl-2014-206260.52}

Rationale All COPD phenotypes have airway neutrophilia but, despite this, bacteria associated infections are common, relate to decline and a significant proportion of patients have persistent airway colonisation. This is suggestive of innate immune dysfunction. In vitro studies have shown reduced neutrophil migratory accuracy in COPD (Sapey, Stockley et al. 2011) however, the ability of the neutrophil to contain bacterial infection upon arrival at a site of infection is poorly understood. Literature regarding the phagocytic ability of neutrophils from patients with COPD is conflicting and inconclusive. It is unclear whether responses change depending on the bacterial species present. We hypothesised that neutrophil phagocytosis during COPD is impaired, predisposing patients to increased inflammation and reduced bacterial clearance.

Methods Blood neutrophils were isolated from stable-state COPD patients and healthy age-matched controls (HC). Phagocytosis of both opsonised (with 10\% pooled COPD serum) and unopsonised pHrodo $^{\mathrm{TM}}$-conjugated Staphylococcus aureus bioparticles (SA, $\mathrm{n}=$

\begin{tabular}{|c|c|c|c|c|c|c|c|}
\hline \multirow[b]{2}{*}{ Respondent's grade } & \multirow[b]{2}{*}{ Number (\%) } & \multirow[b]{2}{*}{ Mean \# of correct answers/20 (SD) } & \multicolumn{3}{|l|}{ Mean level of confidence } & \multicolumn{2}{|c|}{ Decision to Pleurodese } \\
\hline & & & For correct answers (/10) & For incorrect answers $(/ 10)$ & All answers (/10) & $\%$ in "Malignant" & $\%$ in "Benign" \\
\hline Consultant & $13(81 \%)$ & $12.8(2.0)$ & 7.1 & 6.1 & 6.7 & $74 \%$ & $21 \%$ \\
\hline Registrar & $3(19 \%)$ & $11.0(0.0)$ & 7.1 & 6.3 & 6.7 & $48 \%$ & $0 \%$ \\
\hline Total / Overall & $16(100 \%)$ & $12.4(1.9)$ & 7.1 & 6.1 & 6.7 & $69 \%$ & $17 \%$ \\
\hline
\end{tabular}




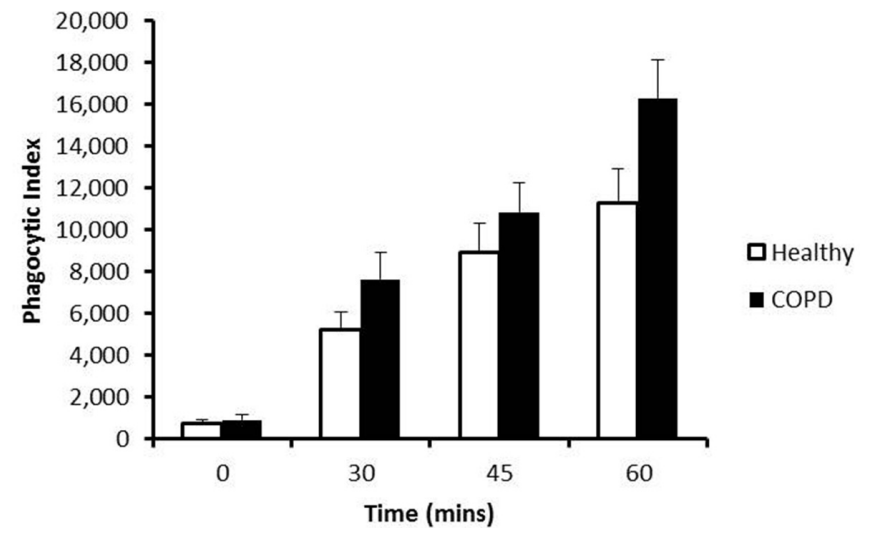

Abstract S46 Figure 1 Phagocytosis of unopsonised SA by blood neutrophils over $60 \mathrm{~min}$

20), or Escherichia coli bioparticles (EC, $\mathrm{n}=10$ ) and fluorescently labelled disease-relevant bacteria, Haemophilus influenzae (HI, $\mathrm{n}$ $=10)$ and Streptococcus pneumoniae (SP, $\mathrm{n}=10$ ) was assessed, at regular intervals over $60 \mathrm{~min}$, using flow-cytometry. Results were confirmed using time-lapse video microscopy.

Results Peak phagocytosis was achieved at $60 \mathrm{~min}$ for unopsonised bacteria and $30 \mathrm{~min}$ for opsonised bacteria. There were no differences in time to peak phagocytosis between bacterial species. Blood neutrophils from patients with COPD and HC displayed similar phagocytic ability, in both percentage of neutrophils with phagocytic activity and the amount of SA, EC, HI or SP ingested (as indicated by MFI) (COPD vs. HC, p > 0.05 for all). This was ubiquitous to both opsonin independent and opsonin-dependant phagocytosis, and was consistent across all time points measured. A typical comparison is shown in figure one, with unopsonised SA data.

Conclusions Phagocytic ability of blood neutrophils from patients with COPD to ingest Staphylococcus aureus, Escherichia coli, Streptococcus pneumoniae and Haemophilus influenzae is not altered compared to age-matched healthy controls. This should be replicated in lung neutrophils to assess whether transmigration to the tissues affects function.

\section{S47 ENHANCED IL-6/CCL3 SIGNALLING IN THE PLASMA OF PATIENTS WITH COPD}

${ }^{1}$ AK Ravi, 'S Khurana, ${ }^{1} \mathrm{~A}$ Banyard, 'I Plumb, ${ }^{1} \mathrm{G}$ Booth, ${ }^{2} \mathrm{M}$ Catley, ${ }^{2} \mathrm{~L}$ Healy, ${ }^{2} \mathrm{E}$ Smith, IJ Vestbo, 'D Singh. 'The University of Manchester, Manchester Academic Health Science Centre, University Hospital of South Manchester NHS Foundation Trust, NIHR South Manchester Respiratory and Allergy Clinical Research Facility, Manchester, UK; ${ }^{2} U C B$, Slough, UK

\subsection{6/thoraxjnl-2014-206260.53}

Rationale IL-6 is a pro-inflammatory cytokine that signals through soluble (sIL-6R/sgp80) and membrane bound (gp80) receptors to promote recruitment of mononuclear cells. IL-6 induces expression of CCL3, a monocytic chemokine. Monocytes are precursors of macrophages and dendritic cells. They can be classified into three subtypes according to surface expression of CD14 (LPS receptor) and CD16 (FcgammaRIII): CD14++CD16-, CD14+CD16+, CD14-CD16++. We measured plasma levels of IL-6, sIL-6R and CCL3 and determined the chemokine receptor expression profile of circulating monocytes in COPD.

Methods 70 COPD patients and 30 healthy controls comprising 15 smokers (S) and 15 healthy non-smokers (HNS) underwent plasma sampling. Levels of IL-6, sIL-6R and CCL3 were
Abstract S47 Table 1

\begin{tabular}{lllll}
\hline & COPD & S & HNS & ANOVA \\
\hline IL-6 $(\mathrm{pg} / \mathrm{ml})$ & $33.7(190.3)$ & $3.8(1.9)$ & $0^{*}$ & $\mathrm{p}<0.0001$ \\
sIL-6R (pg/ml) & $5338(850.3)$ & $4453(613.2)$ & $4853(856.8)$ & $\mathrm{p}=0.0005$ \\
CCL3 $(\mathrm{pg} / \mathrm{ml})$ & $74.8(111.9)^{* *}$ & $0^{*}$ & $0^{*}$ & - \\
\hline
\end{tabular}

Data expressed as mean (SD) * < lower limit of quantification of the assay ${ }^{*} \mathrm{CCL} 3$ levels registered above the assay's lower limit of quantification in 7/70 COPD patients.

determined by multiplex analysis (MSD) of plasma. Multi-colour flow cytometry was performed on whole blood obtained from 32 COPD patients, $8 \mathrm{~S}$ and $8 \mathrm{HNS}$ to measure surface expression levels of chemokine receptors CCR1, CCR2, CCR7, CXCR1 and CX3CR1 on CD14++CD16-, CD14+CD16+ and CD14-CD16++ monocytes.

Results COPD patients had the greatest levels of IL-6 and sIL6R. CCL3 was not detected in any controls, but was present in a subset of COPD patients.\% surface expression of the CCL3 receptor CCR1 measured on CD14++CD16- monocytes of COPD patients was greater than those of HNS $(p=0.04)$. There were no significant differences in expression levels of other chemokine receptors.

Conclusion We report evidence of enhanced IL-6 signalling in the plasma of COPD patients and increased plasma CCL3 in a subset of individuals from this disease group. Furthermore, there was increased CCR1 expression on COPD monocytes. Enhanced IL-6 may co-ordinate the mononuclear component of the inflammatory response in COPD.

\section{S48 AIR POLLUTION PARTICULATE MATTER PROMOTES DC MATURATION AND ENHANCES THEIR STIMULATION OF CD8 LYMPHOCYTE RESPONSES}

TR Ho, PE Pfeffer, E Mann, FJ Kelly, NC Matthews, CM Hawrylowiz. King's College London, London, UK

\subsection{6/thoraxjnl-2014-206260.54}

Background High levels of ambient urban particulate matter (UPM), a component of air pollution, are associated with respiratory tract infections and exacerbations of airways diseases. Dendritic cells (DCs) exposed to inhaled UPM orchestrate the resulting immune response. We have previously shown that UPM-stimulation of DCs results in enhanced proliferation of naïve CD4 lymphocytes but decreased priming of IFN $\gamma$-producing CD4 lymphocytes. These CD4 lymphocytes are important in anti-viral immune responses; however, Tc1 CD8 lymphocytes have more direct anti-viral action. In this research we have studied the effect of UPM on DC priming of CD8 lymphocytes. Methods CD1c peripheral blood DCs were isolated, cultured in the presence/absence of UPM stimulation, with GM-CSF or in medium alone. DC expression of CD83, CCR7, CD40 and MHC Class I were measured by flow-cytometry at $24 \mathrm{~h}$. Pretreated DCs were also cultured with naïve CD8 lymphocytes in

Abstract S48 Table 1 Effect of UPM stimulation of DCs upon naïve CD8 lymphocyte response in MLR at day 5. Median (Inter-Quartile Range) TNF $\alpha$, IFN $\gamma$ and IL-13 production

\begin{tabular}{lllll}
\hline & Control & UPM & GM-CSF & UPM + GM-CSF \\
\hline TNF $\alpha$ (pg/ml) & $77.6(60.2-256)$ & $149(72.3-853)$ & $904(148-1425)$ & $2411(434-4869)$ \\
IFN $\gamma(\mathrm{pg} / \mathrm{ml})$ & $84.5(44.9-195)$ & $225(73.6-1537)$ & $1009(66.25-1477)$ & $2343(189-8726)$ \\
$\mathrm{IL}-13(\mathrm{pg} / \mathrm{ml})$ & $25.9(5.88-106)$ & $59.0(25.3-335)$ & $939(45.8-984)$ & $638(74.6-1266)$ \\
\hline
\end{tabular}

